\title{
Annual research review: the nature and classification of reading disorders - a commentary on proposals for DSM-5
}

\author{
Commented by: Ana Luiza Gomes Pinto Navas ${ }^{1}$
}

Snowling MJ, Hulme C. Annual research review: the nature and classification of reading disorders - a commentary on proposals for DSM-5. J Child Psychol Psychiatry. 2012;53(5):593-607.

The discussions on the revision for the new edition of the Diagnostic and Statistical Manual of Mental Disorders $(\mathrm{DSM}-\mathrm{V})^{(1)}$ are taking place worldwide by researchers, clinicians, politicians, social organizations and other stakeholders. The DSM meets the guidelines for the diagnostic criteria for the classification of many communication and neurodevelopmental disorders. The changes proposed by various expert committees were presented to the scientific community, as well as general society, which in turn sent their comments. These changes, scheduled to be published in May 2013, can be accessed on the website of the American Psychiatric Association $^{(1)}$. One point of great debate at this stage of review is about the category of "learning disorders" that, in addition to changes in diagnostic criteria, also presents a new proposal for classification of disorders of reading and writing.

The article commented here, written by two renowned researchers in the field of Psychology of Reading, presents a critical review that is both actual and relevant to this debate, based on scientific evidence.

The first interesting point made is the justification for including reading and writing disorders on a manual of mental disorders. These disorders are commonly associated with other neurodevelopmental and psychiatric disorders including the presence of varying levels of anxiety and depression. Thus, the authors emphasize the importance of professionals working in the mental health field to pay attention to the identification of cases of reading disorders to take an integral approach, complementary to the educational support that these children need.

According to this article, there are essentially two types of reading disorders: severe difficulties with the decoding of words, or severe difficulties with reading comprehension. The proposed classification in the DSM-V should recognize the continuity between language and reading/writing disorders, as well as the comorbidity with other disorders that greatly increase the risk for developing a learning disorder. A positive change in the new classification would be the dissociation of difficulties in decoding and reading comprehension. However, at this stage, the Manual includes disorders of reading comprehension within the category of "language disorders".

The category of "neurodevelopment disorders" includes

(1) Undergraduate Program in Speech-Language Pathology and Audiology, Faculdade de Ciências Médicas da Santa Casa de São Paulo - FCMSCSP São Paulo (SP), Brazil.

Correspondence address: Ana Luiza Gomes Pinto Navas. R. Doutor Cesário Mota Júnior, 61, $8^{\circ}$ andar, Vila Buarque, São Paulo (SP), Brasil, CEP: 01221-020.

E-mail: ana.navas@fcmsantacasasp.edu.br "learning disorders" and "communication disorders", among others, identified in early childhood education. Among the specific learning disorders are dyslexia, dyscalculia, and disorder of written expression.

Unlike other definitions of associations and experts, the phonological deficit hypothesis is not mentioned on the DSM-V. The development of reading involves mapping of phonological into orthographic representation units. The ease or difficulty of this correspondence depends, among other factors, on the language being learned ${ }^{(2)}$. In languages such as Brazilian Portuguese, which has a transparent system, the process is faster, but the predictive factors for dyslexia, for example, are the same as other less transparent orthographies. The deficit in phonological processing has been universally and persistently linked to these frames throughout the lifespan. The authors emphasize how important it is to make the term explicit, primarily, that this deficit could be identified even before the child starts the process of literacy, promoting the development of early intervention programs. The scientific community, in particular in the area of Speech-Language Pathology, has proposed to include this information as a criterion for early diagnosis.

Another important aspect discussed by the authors is the need for inclusion in the DSM-V of the difficulties in reading comprehension. Many children and young people have severe difficulties with reading comprehension despite a proper decoding. There are many aspects involved in understanding, from vocabulary and morphosyntax to cognitive functions, such as attention and memory, and the ability to make inferences. In addition, difficulties in reading comprehension may be related to other disorders such as Attention Deficit and Hyperactivity Disorder (ADHD) and Autism Spectrum Disorder. In these cases, the comprehension difficulties are secondary to changes in attentional processes and pragmatic functions, respectively. The suggestion of the authors is that these changes in reading comprehension are included in the category "language disorders".

The authors discuss yet another situation that deserves attention in this process of revision of the DSM. The relationship between reading difficulties and "communication disorders", in particular the "language disorders", i.e., children with developmental disorders of language and communication are at risk for developing reading and writing problems ${ }^{(3)}$. On the other hand, it is important to recognize the protective role of language skills for learning disorders. Several studies indicate that a good language development is the foundation 
for successful intervention in cases of dyslexia.

The current proposed revision to the DSM-V presents the distinction between language disorders, Specific Language Impairment (SLI), Phonological Disorder, and Social Communication Disorder (associated with pragmatic difficulties). However, the characterization of the impact of such language disorders on reading and writing is complex. It is known that many of these disorders overlap, and there is also described comorbidities between SLI and dyslexia, for example, and even more serious is the small number of children with the diagnosis of SLI who are identified as learning disorders because of the difficulties in reading comprehension.

The evidence found on the relationship between speech disorders and/or language difficulties and reading and writing, attest to the limited understanding of these relationships within a categorical approach, and instead show the need for a multidimensional perspective to characterize these disorders. There are common risk factors for language disorders and reading, which points to the existence of a "spectrum" of disorders of reading, with varying degrees of influence among the risk factors.

The article also addresses the growing evidence of comorbidity between reading disorders and other neurodevelopmental disorders ${ }^{(4)}$. For example, the most well studied association between dyslexia and ADHD should be mentioned in the manual, as well as developmental coordination disorder (DCD) and dyscalculia. The proposed revision to the DSM-V recognizes the usefulness of a dimensional approach between various mental disorders.

In particular, the relationship between dyslexia and ADHD is of great clinical importance, not only in terms of the difficulties imposed for clinical diagnosis and for treatment decisions, and educational adaptations. When there is an association between the two disorders, the academic performance is significantly worse than when dyslexia or ADHD appear as a single disorder. Moreover, often a single diagnosis is privileged and as a consequence the treatment may focus on one specific aspect, attention or reading.

A study ${ }^{(5)}$ described in this article compared the performance of children with ADHD or dyslexia with a group where the two features appeared as comorbidity (ADHD + dyslexia). The results show that temporal perception difficulties were found only for the ADHD group, whereas changes in phonological processing were found exclusively in the group with dyslexia. The Dyslexia+ADHD group had the worst performance, and showed an additive effect of the difficulties, probably mediated by a common factor, deficits in processing speed. These results are corroborated by clinical studies of genetic basis, pointing to common risk factors between dyslexia and ADHD, particularly with regard to the processing speed component ${ }^{(6)}$.

In summary, this paper presents points of agreement and disagreement with the changes so far made by the Commit- tee for the Revision of the Manual DSM-V. Among the few points that deserve our consideration, the authors suggest, for example, that:

- the deficit in phonological processing is explained as a criterion for diagnosis in cases of specific reading disorder (dyslexia), mainly because of its importance for early diagnosis;

- the "disorder of reading comprehension" is understood as related, but independent of the diagnostic criteria for dyslexia;

- the "disorder of written expression" is differentiated from specific writing disorder (dysorthography), since in the first case aspects of language development are affected and in the second, the difficulty lies in the encoding process at the word level, with involvement of phoneme-grapheme correspondence;

- the "language disorders" and "reading disorders" are understood in a dimensional model so that the language difficulties at the phonological system are considered risk factors for deficits in decoding words (dyslexia). On the other hand, more comprehensive difficulties in oral language (including semantic and syntactic aspects) are considered risk factors for disorders of reading comprehension.

Finally, the article presents a comprehensive and broadbased international scientific literature on the relationship between neurodevelopmental disorders, communication disorders and learning disorders. With this article, the authors intended to alert the entire scientific community to engage more directly in the review process for DSM-V, contributing to these discussions extremely relevant for clinical work, and especially to the establishment of policies public monitoring and intervention for these disorders.

\section{REFERENCES}

1. American Psychiatric Association. DSM-5 Development [Internet]. 2011 [cited 2012 Aug 27]. Available from: http://www.dsm5.org/Pages/ Default.aspx.

2. Ziegler JC, Goswami U. Reading acquisition, developmental dyslexia, and skilled reading across languages: a psycholinguistic grain size theory. Psychol Bull. 2005;131(1):3-29.

3. Bishop DV, Snowling MJ. Developmental dyslexia and specific language impairment: same or different? Psychol Bull. 2004;130(6):858-86.

4. Willcutt EG, DeFries JC, Pennington BF, Olson RK, Smith SD, Cardon LR. Genetic etiology of comorbid reading difficulties and ADHD. In: Plomin R, DeFries JC, Craig IW, McGuffin P (Eds.). Behavioral genetics in a postgenomic era. Washington, DC: American Psychological Association; 2003. p. 227-246.

5. Gooch D, Snowling M, Hulme C. Time perception, phonological skills and executive function in children with dyslexia and/or ADHD symptoms. J Child Psychol Psychiatry. 2011;52(2):195-203.

6. Willcutt EG, Pennington BF. Comorbidity of reading disability and attention-deficit/hyperactivity disorder: differences by gender and subtype. J Learn Disabil. 2000;33(2):179-191. 